\title{
Morphology of the temporalis muscle focusing on the tendinous attachment onto the coronoid process
}

\author{
Sun Kyoung $\mathrm{Yu}^{1,2}$, Tae-Hoon Kim${ }^{1}$, Kwang Yeol Yang ${ }^{1}$, Christopher J. Bae ${ }^{2}$, Heung-Joong Kim ${ }^{1}$ \\ ${ }^{1}$ Department of Anatomy and Orofacial Development, College of Dentistry, Chosun University, Gwangju, Korea, ${ }^{2}$ Department of Anthropology, \\ University of Hawai'i at Manoa, Honolulu, HI, USA
}

\begin{abstract}
The temporalis muscle is usually described as a single layer originating at the temporal line, converging to a tendon, and inserting onto a narrow site of the coronoid process. However, recent studies have shown that the temporalis muscle can be divided into two or three separate segments and the distal attachment continues inferiorly beyond the coronoid process. Therefore, the aims of this study were to analyze the morphology of the temporalis muscle focusing on the tendinous attachment onto the coronoid process and to provide educational values. The temporalis muscle was carefully dissected in 26 cadavers and classified based on the muscle fascicle direction. Each divided part was sketched and measured based on bony landmarks to elucidate its tendinous insertion site onto the coronoid process, and the results obtained were reviewed through the literature. The temporalis muscle ends at two distinct terminal tendons with wider insertion sites than usually presented in textbooks and atlases and separates into two parts that combine to act as a single structural unit. The superficial part is a large fan-shaped muscle commonly recognized as the temporalis muscle. This converges infero-medially to form the superficial tendon and the lateral boundary of the retromolar triangle. Meanwhile, the deep part is a narrow vertically oriented rectangular muscle that converges postero-laterally to form the deep tendon and the medial boundary of the retromolar triangle. These results indicate that understanding the temporalis muscle's insertion site onto the coronoid process will be useful clinically with educational values during surgical procedures.
\end{abstract}

Key words: Temporalis muscle, Coronoid process, Superficial temporalis tendon, Deep temporalis tendon, Retromolar region

Received April 14, 2021; Revised June 8, 2021; Accepted June 18, 2021

\section{Introduction}

The temporalis muscle (TM) is composed of a single layer within the temporal line of the parietal bone and attaches to the coronoid process [1-4]. In major textbooks and atlases, it

\section{Corresponding author:}

Heung-Joong Kim (iD

Department of Anatomy and Orofacial Development, College of Dentistry, Chosun University, Gwangju 61452, Korea

E-mail: hjbkim@chosun.ac.kr is usually illustrated simply as originating at a wide attachment site, converging to the form of a tendon, and inserting onto a narrow site of the coronoid process [5-7]. However, recent studies have shown that the distal tendon of the TM with a wider and lower attachment site continues inferiorly to the retromolar triangle and forms the pterygomandibular depression $[7,8]$. This extended attachment site of the TM can provide a better balance on the forces produced by the muscles [7]. In addition, it is used as a landmark for inferior alveolar nerve block anesthesia, third molar extraction, and determining posterior denture flange, and is also used for direct temporalis tendon injections and temporalis tendon 
transfers in plastic surgery [7, 9-12]. These various clinical applications in the mandible require accurate knowledge of the morphology of the TM including its tendinous attachment.

Following muscle fiber direction is important because it determines the force vectors that it can exert [2]. Currently, the TM is divided into three parts according to fiber direction: anterior (orbital or vertical fibers), middle (temporal or oblique fibers), and posterior (horizontal fibers) [13-15]. According to electromyographic analysis, these fiber directions are responsible for the mandible protrusion, rotation, and retraction, respectively $[13,14,16]$. Studies using Sihler's staining, which allows observation of the nerve distribution within the muscles without dissection, also revealed that the three parts of the temporal muscle were each innervated respectively by the anterior deep temporal nerve, the middle deep temporal nerve, and the posterior deep temporal nerve [15].

Study of the development of the TM in the human fetus found that it extends inferiorly to two muscle fascicles onto the coronoid process: one that inserts into the anterior border of the mandibular ramus and the other that inserts into the medial surface of the ramus [17]. Another study of the TM confirmed the presence of two separate tendons: (1) a superficial tendon attached to the anterior border of the mandibular ramus; and (2) a deep tendon attached to the temporal crest of the medial surface of the ramus $[8,13$, 18]. Further, a histological study found that, in contrast to the superficial part, the deep part includes numerous nerve structures that are similar to the superior belly of the lateral pterygoid muscle [1]. Thus, the presence of these two distinct terminal tendons could raise the possibility that the TM is divided into two layers or parts. These different studies indicate clearly that the morphology of the TM is not as simple as previously understood.

Studies have shown clearly that the TM is not a single layer and that its morphology is often confused because of various nomenclature and differently classified muscle parts [19]. For example, the anterolateral and anteromedial muscle bundles of the TM are variously named, such as the sphenofrontal part of the temporalis [20] and the first layer of the zygomaticomandibularis [21], and the deep sphenoid part of the temporalis [20], the medial portion of the temporalis [22], the second layer of the zygomaticomandibularis [21], and the accessory head of the temporalis [23], respectively. Furthermore, Shankland et al. [24] and Dunn et al. [25] reported these as new muscles, and were given the name "pre-anterior belly" of the TM and "sphenomandibularis." Because of the inconsistency in describing the TM, particularly the lateral and medial parts, it is necessary to evaluate more clearly the anatomy and action on the superficial layer or lateral portion and the deep layer or medial portion of the TM.

Therefore, the aims of this study were to analyze the morphology of the TM focusing on the tendinous attachment in order to identify how each part of the TM fascicle attaches onto the coronoid process, and to provide educational values.

\section{Materials and Methods}

We dissected the TM in 26 embalmed cadavers that were donated for educational purposes to the Department of Anatomy, Chosun University School of Medicine in Gwangju, Republic of Korea. They comprised 18 males and 8 females whose age at death ranged from 40 to 97 years, with a mean age of 64.9 years. We chose these 26 specimens (14 right and 12 left) because they displayed no obvious signs of damage to the TM and the mandible regardless of any morphological changes resulting from any tooth loss. This study followed in accordance with the ethical principles for medical research involving human subjects, outlined in the Declaration of Helsinki of 1975.

To elucidate the tendinous attachment and insertion site of the TM onto the coronoid process, the skin and subcutaneous tissues of each hemiface were first removed to reveal the superficial temporal fascia and the masseter muscle. Following the methods described in Schön Ybarra and Bauer [2] and Geers et al. [1], the masseter muscle and the zygomatic arch were removed in turn. In that way, the origin of the TM and the mandible blocks that contain the entire TM could be identified. The retromolar pad was removed via a frontal approach without damaging the temporal tendon and then carefully dissected with the aid of a surgical microscope (OPMI-FC; Carl Zeiss, Oberkochen, Germany). After removing the superficial and deep temporal fasciae, the TM was dissected and separated based on the direction of the muscle fascicle. After each divided part was sketched, its insertion site that presented repeatedly were analyzed. And the results obtained were reviewed through the literature.

To analyze the portion of each part attached to the coronoid process, the two divided tendons were measured based on bony landmarks such as the inferior border of the mandible, the mandibular notch, and the lingula that is a bone 


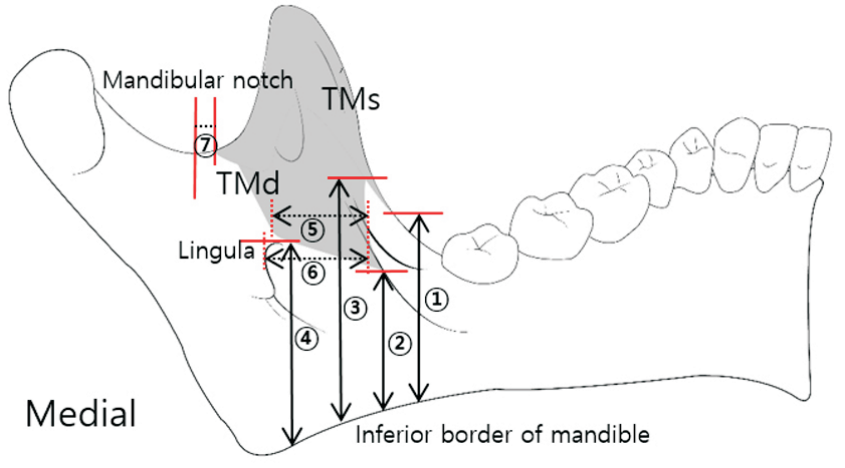

Fig. 1. Diagram showing the dimensions measured the two separate parts of the temporalis muscle attached onto the coronoid process based on the bony landmarks. (1), height of the ending point of the superficial part of the temporalis muscle (TMs) from the inferior border of the mandible; (2), height of the ending point of the deep part of the temporalis muscle (TMd) from the inferior border of the mandible; (3), height of the separating point of the two parts from the inferior border of the mandible; (4), height of the lingula from the inferior border of the mandible; (5), width of the TMd from the anterior border of the mandibular ramus; (6), width of the lingula from the anterior border of the mandibular ramus; (7), distance of the end point of the posterior border of the TMs based on the mandibular notch.

structure anterior to the mandibular foramen that serves as the entrance of the inferior alveolar neurovascular bundle. It was measured with a digital caliper (Mitutoyo, Kawasaki, Japan) by the height from the inferior border of the mandible to their lowest points, the width from the anterior border of the mandibular ramus in the mandibular medial surface, and the end point of the posterior border based on the mandibular notch (Fig. 1). The superior angle, where the superficial and deep tendons separated, was measured in the photograph taken from the anterior direction of the mandibular ramus as in Fig. 2. Two observers performed the measurements twice on different days following the same method. At this time, the measurement difference between both observers showed no significant differences in any of the measurement items. Thus, the subsequent analyses used the average of the values measured between both observers. Statistical analysis was performed utilizing IBM SPSS version 22.0 (IBM Corp., Armonk, NY, USA). One-way ANOVA was used to detect significant differences according to the height from the inferior border of the mandible to each measurement point. A post-hoc comparison on Scheffe was then performed. All measurements are presented as mean \pm SD values, and the significance criterion was set at $P<0.05$.
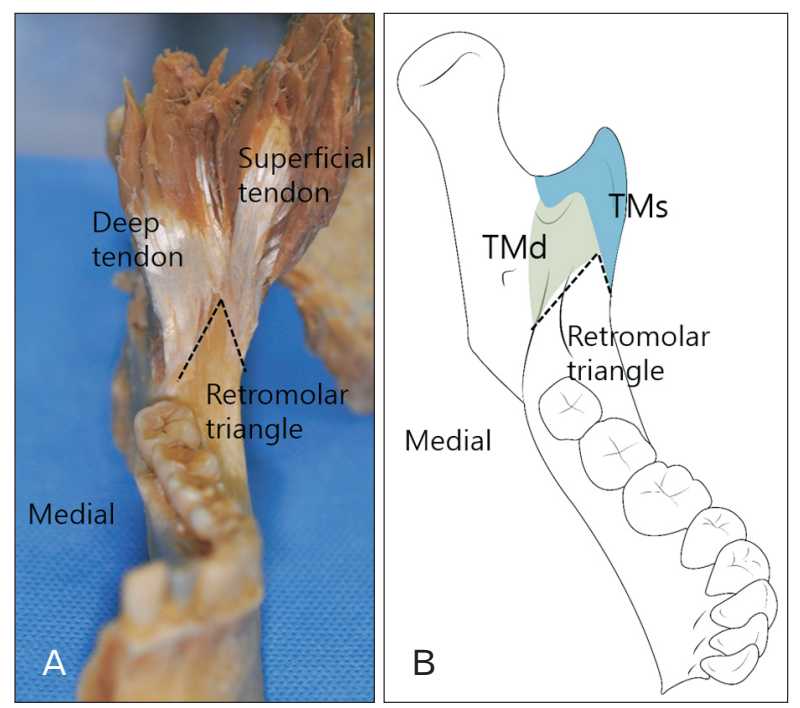

Fig. 2. Photograph (A) and schematic (B) illustrating the two distinct terminal tendons of the temporalis muscle that formed the boundary on both sides of the retromolar triangle. The blue and green colors indicate the dimensions of the TMs and TMd onto the coronoid process, respectively. TMs, superficial part of the temporalis muscle; TMd, deep part of the temporalis muscle.

\section{Results}

The TM separates and attaches as two strong tendons on the lateral and medial sides of the coronoid process: (1) a superficial tendon and (2) a deep tendon (Fig. 2). The superficial part of the TM is the large fan-shaped TM that is often described in many textbooks and anatomical atlases. It also originates in the inferior temporal line and temporal fossa, descends into the superficial tendon infero-medially, and inserts at the apex, anterior, and posterior margins on the lateral and medial surfaces of the coronoid process (Figs. 3, 4). This superficial tendon descends into the external oblique line along the anterior border of the mandibular ramus and stops close to the third molar; thus forming the lateral boundary of the retromolar triangle on the buccal side (Fig. 2). The lowest point of this tendon is located at a mean height of $3.29 \pm 0.55 \mathrm{~cm}$ from the inferior border of the mandible, which was slightly below the lingula (Table 1). Its end point at the posterior border stops anteriorly at a mean of $0.33 \pm 0.15$ $\mathrm{cm}$ based on the mandibular notch.

As other research has shown $[1,2]$ the present study reconfirms that the deep part of the TM is located on the medial side of the superficial part of the TM. The deep segment arises from the anterior and posterior ridge of the pyramidal-shaped process on the infratemporal crest of the 

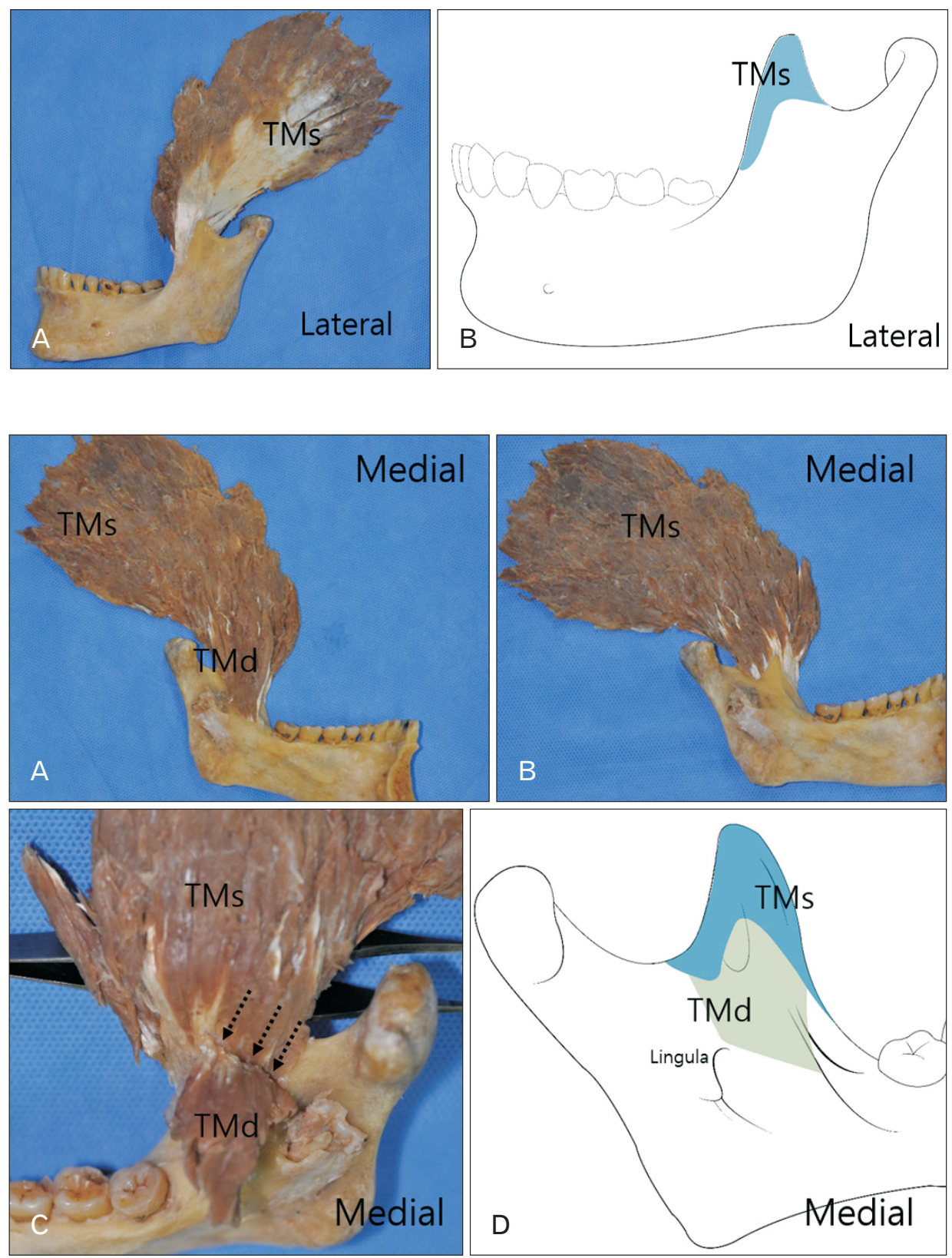

Fig. 3. Photograph (A) and schematic (B) showing the dimensions of the TMs attached onto the coronoid process on the lateral side of the mandible. TMs, superficial part of the temporalis muscle.
Fig. 4. Photographs $(\mathrm{A}-\mathrm{C})$ and schematic (D) showing the dimensions of the TMs and TMd attached onto the coronoid process on the medial side of the mandible. The dotted arrows indicated that the muscle fibers of two separate parts of the temporalis muscle were intermingled in the posterior side. TMs, superficial part of the temporalis muscle; TMd, deep part of the temporalis muscle.

Table 1. Dimension of the two separate parts of the temporalis muscle attached onto the coronoid process based on the bony landmarks

\begin{tabular}{lcccl}
\hline \multicolumn{1}{c}{ Measurement item } & Minimum $(\mathrm{cm})$ & Maximum $(\mathrm{cm})$ & Mean \pm SD $(\mathrm{cm})$ & $6.51 \pm 0.80$ \\
\hline Height of apex of coronoid process & 5.30 & 8.45 & $3.29 \pm 0.55$ & 1 \\
Height of ending point of TMs & 2.20 & 4.32 & $3.05 \pm 0.36$ & 0.50 \\
\hline Height of ending point of TMd & 2.51 & 3.65 & $4.84 \pm 0.74$ & 0.74 \\
Height of separating point of TMs and TMd & 3.58 & 6.67 & $3.42 \pm 0.64$ & 0.52 \\
Height of lingula & 2.13 & 4.30 & $3.43 \pm 0.29$ & 1 \\
Width of mandibular ramus & 2.94 & 3.91 & $1.46 \pm 0.41$ & 0.43 \\
\hline Width of TMd & 0.77 & 2.48 & $1.51 \pm 0.47$ & 0.44 \\
\hline Width of lingula & 0.66 & 2.56 & \\
\hline
\end{tabular}

Each height was measured from the inferior border of the mandible, and each width was measured from the anterior border of the mandibular ramus. The ratio of height was based on a mean height of apex of the coronoid process from the inferior border of the mandible, and the ratio of width was based on a mean width of the mandibular ramus from the anterior border of the mandibular ramus. TMs, superficial part of the temporalis muscle; TMd, deep part of the temporalis muscle. 
greater wing of the sphenoid bone that lies inside the anterior roof of the infratemporal fossa and the pterygopalatine fossa. This narrow and long rectangular muscle descends slightly postero-laterally, which contrasts with the superficial part of the TM, forms the deep tendon and attaches only to the medial surface of the coronoid process. This deep tendon runs vertically along the lingual margin of the temporal crest, forms the medial boundary of the retromolar triangle on the lingual side, and stops closer to the third molar than the superficial tendon (Figs. 2, 4). In particular, while the muscle fibers of the deep part of the TM was removing, these two tendons could be clearly distinguished when examined from the anterior direction of the mandibular ramus, while their tendinous structures were not as clearly delineated when viewed from the posterior direction (Fig. 4). The lowest point of this tendon is located at a mean height of $3.05 \pm 0.36 \mathrm{~cm}$ from the inferior border of the mandible and lies below the insertion site of the superficial part of the TM (Table 1). It has a mean width of $1.46 \pm 0.41 \mathrm{~cm}$ and stops ahead of the lingula (Table 1). The mean angle of the superior angle, where the superficial and deep tendons separated, is $28.9^{\circ} \pm 3.77^{\circ}$ ranged from $22^{\circ}$ to $38.5^{\circ}$. These two tendons are separated at a mean height of $4.84 \pm 0.74 \mathrm{~cm}$ from the inferior border of the mandible. This is higher than the end point of each tendon, resulting in a retromolar fossa of the triangular shape where the minor salivary gland and the retromolar pad are located.

\section{Discussion}

The TM is a large fan-shaped muscle that has a wide attachment site, a large volume, and serves as one of the essential masticatory muscles that perform elevation, retraction, and unilateral contraction of the mandible $[5,6,13]$. Although these complicated and delicate masticatory action, in many textbooks and anatomical atlases the TM is often described simply as a single layer muscle that originates from the inferior temporal line and temporal fossa and inserts into the coronoid process [5-7]. However recently, Benninger and Lee [7] suggested that the distal lateral tendon and distal medial tendon head of the TM should be marked and illustrated beyond the coronoid process and as far as the retromolar fossa to represent the segment most accurately. In addition, recent studies have shown that the TM can be divided into two or three separate segments $[1-3,11,14]$. Therefore, the purpose of this study was to analyze the morphology of the
TM focusing on the tendinous attachment onto the coronoid process.

Here, we found that the TM was also attached to two strong terminal tendons and divided into a superficial layer and a deep layer. This is in agreement with some previous studies $[1,2]$. The superficial part of the TM represents what is commonly known as the TM and constitutes the main part as a large fan-shaped muscle. It forms the superficial tendon that is positioned along the anterior border of the mandibular ramus to the lingula level and forms the lateral border of the retromolar triangle. Because the buccal nerve passes over the surface of this superficial tendon, care should be taken when making any incision in the retromolar area [26]. Interestingly, the size of this superficial part of the TM decreases with age [27], and so further investigation is required into the effect of tooth loss on both the dimensions of the TM and mastication more generally.

There have been questions of whether the anterolateral muscle bundle of the TM is an independent muscle by itself [19-21, 24]. This muscle originates from the posterior region of the orbital part of the zygomatic bone and the outer lateral region of the greater wing of the sphenoid bone, and inserts directly into the anterior border of the mandibular ramus $[7,19]$. In the present study, this muscle forms part of the anterior portion of the superficial part of the TM and the superficial tendon. Akita et al. [19] reports that this muscle is distributed by the anterior deep temporal nerve. Therefore, it is more appropriate to recognize the anterolateral muscle bundle of the TM as part of the anterior portion of the main part of the TM. And it is thought that extended distal tendon attachment of this muscle provides stronger action of elevation and protrusion to the mandible.

The deep belly or medial portion of the TM is located on the medial side of its main part. This area originates from the pyramidal-shaped process on the infratemporal surface of the greater wing of the sphenoid bone and inserts into the temporal crest of the medial surface of the ramus $[1,2]$. Dunn et al. [25] concluded that the deep belly is an independent muscle named as "sphenomandibularis" distinct from the classic description of the TM. However, here we found that the deep temporal fascia covers both the superficial part and the deep part of the TM. Because this muscle is distributed by the anterior-most twigs of the anterior deep temporal nerve [28], Akita et al. [19] considers that this anteromedial muscle bundle is closely related to the anterior portion of the main part of the TM. It is therefore reasonable to assume 
that the deep part of the TM is simply one part of a single muscular complex that is combined with its superficial part, not an independent muscle.

Previous studies found that the deep part of the TM had a mean length of $5.2 \mathrm{~cm}$ and mean width of $1.4 \mathrm{~cm}$, and that it was situated inferior to the superficial part on the anterior side and ahead of the mandibular foramen on the posterior side $[1,8,12,25]$. In the present study, the deep part was found to be a vertically oriented rectangular muscle with a mean width of $1.4 \mathrm{~cm}$ and attached ahead of the lingula, as can also be seen from the results of Akita et al. [19]. Thus, when the deep tendon is damaged due to failure of the inferior alveolar nerve blocks in the pterygomandibular space around the mandibular foramen behind the lingula [29], the patient may experience severe pain and difficulty in opening and closing the mandible. The deep tendon forms the medial boundary of the retromolar triangle and stops at a mean height of about $3 \mathrm{~cm}$ from the inferior border of the mandible inferior to the superficial tendon. This means that the temporalis tendon can be used as a reference landmark for surgical procedures in the retromolar region, including determination of the posterior denture flange and reduction of temporomandibular joint dislocations. The finding reported here could also be useful during manual palpation for direct injections into the temporalis tendon.

Although the deep and superficial parts of the TM are a single muscular complex covered by the deep temporal fascia, the deep part needs to be separated from the superficial part to better understand the muscle action. This is because the deep part has a distinct origin and insertion sites and its muscle fibers descend slightly postero-laterally, in contrast to the superficial segment that descends infero-medially. This could mean that the superficial part operates unilateral contraction on the working side while the deep part provides unilateral contraction on the balancing side. Since lateral movements on the bilateral temporomandibular joint are much more complicated and delicate than the movements of opening and closing the mouth, it apparently requires the subdivision of the TM according to the direction of the muscle fascicle forming the tendon for understanding these complicated masticatory movements. However, as a limitation of this study, which dissected a small number of cadavers, the muscle action of these two parts could not be confirmed. In addition, these two parts were found to be intermingled on the posterior side without any special structure dividing them. Thus, further studies are needed to examine action of the two parts of the TM in detail using electromyographic analysis and 3 dimensional reconstruction.

In conclusion, the TM ends at two distinct terminal tendons with wider insertion sites than usually presented in major textbooks and atlases. Thus, it is clear that the temporalis tendon can be separated into two separate parts that combine to act as a single structural unit. The superficial part is a large fan-shaped muscle commonly recognized as the TM that converges infero-medially to form the superficial tendon and the lateral boundary of the retromolar triangle. Meanwhile, the deep part is a narrow vertically oriented rectangular muscle that converges postero-laterally to form the deep tendon and the medial boundary of the retromolar triangle. These results on the anatomical morphology of the $\mathrm{TM}$ as related to its insertion site onto the coronoid process will be useful clinically with educational values during surgical procedures in the retromolar region, such as extraction, anesthesia, and reduction of temporomandibular joint dislocations.

\section{ORCID}

Sun Kyoung Yu: https://orcid.org/0000-0003-0801-1663

Tae-Hoon Kim: https://orcid.org/0000-0002-5676-4208

Kwang Yeol Yang: https://orcid.org/0000-0002-8320-3027

Christopher J. Bae: https://orcid.org/0000-0003-2972-7230

Heung-Joong Kim: https://orcid.org/0000-0003-1636-3669

\section{Author Contributions}

Conceptualization: SKY, CJB. Data acquisition: THK, KYY. Data analysis or interpretation: THK, KYY, HJK. Drafting of the manuscript: SKY, THK. Critical revision of the manuscript: CJB, HJK. Approval of the final version of the manuscript: all authors.

\section{Conflicts of Interest}

No potential conflict of interest relevant to this article was reported.

\section{Acknowledgements}

The authors wish to thank the individuals who donated their bodies for the advancement of medical education and science. This manuscript was completed while the first au- 
thor was a Visiting Scholar in the Center for Korean Studies, University of Hawai'i at Manoa (2020).

This research was supported by Basic Science Research Program through the National Research Foundation of Korea (NRF) funded by the Ministry of Education (No. 2016R1D1A1B02008797) and by the Ministry of Science and ICT (No. 2017R1A1A3A04069265).

\section{References}

1. Geers C, Nyssen-Behets C, Cosnard G, Lengelé B. The deep belly of the temporalis muscle: an anatomical, histological and MRI study. Surg Radiol Anat 2005;27:184-91.

2. Schön Ybarra MA, Bauer B. Medial portion of M. Temporalis and its potential involvement in facial pain. Clin Anat 2001;14:25-30.

3. Sedlmayr JC, Kirsch CF, Wisco JJ. The human temporalis muscle: superficial, deep, and zygomatic parts comprise one structural unit. Clin Anat 2009;22:655-64.

4. Lee JY, Kim JN, Kim SH, Choi HG, Hu KS, Kim HJ, Song WC, Koh KS. Anatomical verification and designation of the superficial layer of the temporalis muscle. Clin Anat 2012;25:176-81.

5. Drake RL, Vogl W, Mitchell AWM, Gray H. Gray's anatomy for students. 2nd ed. Philadelphia: Churchill Livingstone; 2010.

6. Schuenke M, Schulte E, Schumacher U. Thieme atlas of anatomy. Head and neuroanatomy. New York: Thieme Stuttgart; 2010.

7. Benninger B, Lee BI. Clinical importance of morphology and nomenclature of distal attachment of temporalis tendon. J Oral Maxillofac Surg 2012;70:557-61.

8. Harn SD, Shackelford LS. Further evaluation of the superficial and deep tendons of the human temporalis muscle. Anat Rec 1982;202:537-48.

9. Bressler HB, Friedman T, Friedman L. Ultrasound-guided injection of the temporalis tendon: a novel technique. J Ultrasound Med 2017;36:2125-31.

10. Owusu Boahene KD. Temporalis muscle tendon unit transfer for smile restoration after facial paralysis. Facial Plast Surg Clin North Am 2016;24:37-45.

11. Palomari ET, Picosse LR, Tobo MP, Isayama RN, da Cunha MR. Sphenomandibular muscle or deep bundle of temporal muscle? Int J Morphol 2013;31:1158-61.

12. Parker NP, Eisler LS, Dresner HS, Walsh WE. Orthodromic temporalis tendon transfer: anatomical considerations. Arch Facial Plast Surg 2012;14:39-44.

13. Kim MK. Head and neck anatomy. 6th ed. Seoul: Dental \& Medical Publishing; 2017.

14. Gaudy JF, Zouaoui A, Bravetti P, Charrier JL, Laison F. Functional anatomy of the human temporal muscle. Surg Radiol Anat 2001;23:389-98.
15. Won SY, Kim DH, Yang HM, Park JT, Kwak HH, Hu KS, Kim HJ. Clinical and anatomical approach using Sihler's staining technique (whole mount nerve stain). Anat Cell Biol 2011;44:17.

16. Blanksma NG, van Eijden TM. Electromyographic heterogeneity in the human temporalis and masseter muscles during static biting, open/close excursions, and chewing. J Dent Res 1995;74:1318-27.

17. Mérida-Velasco JR, Rodríguez-Vázquez JF, De La Cuadra C, Mérida-Velasco JA, Jiménez-Collado J. The course of the buccal nerve: relationships with the temporalis muscle during the prenatal period. J Anat 2001;198(Pt 4):423-9.

18. Logan BM, Hutchings RT, Reynolds PA, McMinn RMH. McMinn's Colour atlas of head \& neck anatomy. 3rd ed. London: Mosby; 2004.

19. Akita K, Sakaguchi-Kuma T, Fukino K, Ono T. Masticatory muscles and branches of mandibular nerve: positional relationships between various muscle bundles and their innervating branches. Anat Rec (Hoboken) 2019;302:609-19.

20. Eisler P. [Die Muskeln des stammes]. In: von Bardeleben K, editor. [Handbuch der anatomie des menschen]. Jena: Gustav Fischer; 1912. pp. 197-220. German.

21. Yoshikawa T, Suzuki T. The lamination of the human masseter- the new identification of $M$. temporalis superficialis, $M$. maxillomandibularis and M. zygomaticomandibularis in the human anatomy. Acta Anat Nippon 1962;37:260-7.

22. Zenker W. [Function of the medial portion of the M. temporalis]. Osterr Z Stomatol 1954;51:550-4. German.

23. Tomo S. Morphological classification of the mastication muscle based on their innervation. Ochanomizu Med J Tokyo 1990;38:57-71. Japanese.

24. Shankland WE 2nd, Negulesco JA, O’Brian B. The “pre-anterior belly" of the temporalis muscle: a preliminary study of a newly described muscle. Cranio 1996;14:106-12.

25. Dunn GF, Hack GD, Robinson WL, Koritzer RT. Anatomical observation of a craniomandibular muscle originating from the skull base: the sphenomandibularis. Cranio 1996;14:97-103.

26. Kim HJ, Park BS, Cho YH, Yu SK. Course of buccal nerve on the anterior border of mandibular ramus related to temporalis tendon. Oral Biol Res 2017;41:236-9.

27. Ranganathan K, Terjimanian M, Lisiecki J, Rinkinen J, Mukkamala A, Brownley C, Buchman SR, Wang SC, Levi B. Temporalis muscle morphomics: the psoas of the craniofacial skeleton. J Surg Res 2014;186:246-52.

28. Shimokawa T, Akita K, Soma K, Sato T. An anatomical study of the muscles innervated by the masseteric nerve. Okajimas Folia Anat Jpn 1999;75:271-80.

29. Khoury JN, Mihailidis S, Ghabriel M, Townsend G. Applied anatomy of the pterygomandibular space: improving the success of inferior alveolar nerve blocks. Aust Dent J 2011;56:112-21. 